\title{
EXPANDIENDO PARADIGMAS, REDISEÑANDO FRONTERAS: LA DIPLOMACIA CULTURAL NORTEAMERICANA Y LA BÚSQUEDA DE UNA COMUNIDAD INTERAMERICANA DE ACADÉMICOS
}

\author{
Claudio González-Chiaramonte* \\ Universidad de Buenos Aires/Argentina \\ Middlebury Collage/USA
}

Resumen: Dentro del marco de su política hacia el liderazgo hegemónico después de la Segunda Guerra Mundial, EE.UU. comenzó a desarrollar iniciativas dirigidas a los campos cultural y académico de América Latina con el objetivo de atraer a los intelectuales y a las elites de la región hacia su proyecto estratégico. El proceso de la Guerra Fría aceleró la emergencia del campo de la diplomacia cultural como un eje paralelo y complementario a la diplomacia tradicional, a la cual estaba subordinada, aunque manteniendo métodos, dinámicas y objetivos específicos. Programas y actividades diversos como el intercambio de estudiantes, intelectuales y líderes; la asistencia técnica, militar, informativa, y de inteligencia; la promoción del idioma inglés y de las industrias de Hollywood y de la TV; entre varias iniciativas, procuraron "ganar los corazones y las mentes" de las sociedades locales. La proyección política de la diplomacia cultural recurrió a agencias federales, universidades, y sociedades filantrópicas para apoyar iniciativas culturales que, más allá de difundir paradigmas científicos y artísticos, también constituyó la exportación de una ideología: la promoción del "sueño americano." La llegada a América Latina de estos programas, instituciones, y funcionarios organizados y financiados por EE.UU. promovió la creación de una red de elites locales constituída por instituciones, programas y académcos que gozaban de mejores condiciones de producción ventajosas a través de su fuerte articulación con el campo cultural norteamericano de referencia. El contacto de este nuevo sector con los "más modernos" paradigmas científicos norteamericanos, así como la inmediata asociación de las diferencias intradisciplinarias emergentes a conflictos políticos locales, internacionales, y de la Guerra Fría - algo poco sorprendente, considerando la poca autonomía del campo cultural local con respecto al poder político - contribuyeron a profundas divisiones dentro de los campos académico y cultural. Sin embargo, lejos de haber tenido consecuencias homogéneas, la

\footnotetext{
* El autor agradece la colaboración de Rockefeller Archive Center, Universidad Torcuato Di Tella, y Fundación Ford.
} 
emergencia de estos "sitios de contacto" trans-nacionales abrió un proceso dinámico de intercambio cultural que superó los objetivos de la diplomacia de EE.UU. Estos "sitios de contacto" de la expansión cultural y política norteamericana constituyen un área fértil para el estudio de no sólo las políticas imperiales, sino también de los múltiples agentes, instituciones, e ideas, nacionales y trans-nacionales, que entraron en contacto a través de ese proceso.

\section{Expanding Paradigms, Redesigning Frontiers: American Cultural Diplomacy and the Quest for an Inter-American Community of Scholars}

Abstract: Within the frame of a policy for hegemonic leadership in post-World War II, the U. S. started to display initiatives aimed at the Latin American academic and cultural fields with the goal of attracting those intellectuals and elites toward its strategic thought and project. The process of the Cold War accelerated the emergence of the field of cultural diplomacy as an axis parallel to and complementary with traditional diplomacy, to which it was subordinated although with its own specific methods, dynamics, and goals. Diverse programs and activities, such as exchange of students, intellectuals, and leaders; intelligence, informational, military, and technical assistance; promotion of the teaching of English as well as of the TV and Hollywood industries, among others, sought to "win the minds and hearts" of the local societies. As a steady axis of this political projection, the U. S. cultural diplomacy recurred to federal agencies, universities, and philanthropic societies to support cultural initiatives that, beyond expanding scientific and artistic paradigms, also constituted the export of an ideology: the promotion of the American dream The arrival of these institutions, programs, and officials organized and financed by the U. S. promoted the creation in the local field of a local elite network constituted by institutions, programs, and professionals with better conditions of production and a strong articulation within the American academic field of reference. The link of this sector to the "more modern" American scientific paradigms, as well as the immediate association of those disciplinary differences to Cold War, local and international political conflicts - a less than surprising fact given the reduced autonomy of the local cultural field with respect to national politics - contributed to the deep division within this field. However, the emergence of these transnational "sites of contact," far from having homogenous consequences, opened a dynamic process of cultural exchange that went beyond the U. S. foreign policy goals. These "sites of contact" of the American cultural expansion constitute a fertile area not only for the study of imperial policies but also of the multiple agents, institutions, and ideas, national as well as trans-national, that met each other through this process. 
En 1961, un informe de la Fundación Rockefeller sobre la creación del Centro Internacional para la Investigación Social Comparada (ICSSR) en el prestigioso Instituto Torcuato Di Tella, en Buenos Aires, Argentina, notaba que la rápida transformación que estaba ocurriendo en América Latina demostraba que los estudios internacionales eran imprescindibles para el adelanto del conocimiento en el campo de las Ciencias Sociales. Sin embargo, el informe también lamentaba que "los recursos humanos y materiales dedicados realmente a tal propósito son, por lo menos, escasos e insuficientes." La situación se volvía aún más seria si se tomaba en cuenta "la carencia de comunicación entre los pocos científicos sociales y centros de investigación latinoamericanos que existen en el área."1

En consecuencia, "un énfasis especial sería dado a los proyectos cooperativos dirigidos a la promoción del desarrollo de la investigación científica en la Argentina y en otros países latinoamericanos, donde tal actividad [era] escasa o inexistente." ${ }^{2}$ Sin embargo, en el caso específico de la Argentina, mientras que el área de Buenos Aires sería servida por el proyecto del ICSSR, "la situación existente [en los campos de] la sociología, de la investigación social y de las ciencias sociales en general en la mayoría de las universidades provinciales haría muy difícil o imposible ampliar tal influencia al 'interior' del país [por] la mayor resistencia a la introducción de investigación social moderna y a aumentar sus standards académico y científico." 3

Debido a que "la investigación social imparcial e independiente de valores ideológicos [era] relativamente nueva en la Argentina y América latina," los funcionarios de la Fundación Rockefeller reconocían que "la Sociología [era] percibida con frecuencia como ideológicamente orientada, incluso cuando los científicos sociales intentaban ser tan imparciales como fuera posible." La estrategia para desarrollar la investigación social en la Argentina contaba con que "el Centro proyectado sería absolutamente independiente de los riesgos de inestabilidad que caracterizaban a instituciones públicas - por ejemplo, las universidades nacionales en America Latina." En un país donde "las actividades de la universidad eran percibidas a menudo como políticas, y dadas las interpretaciones más fantásticas," la Fundación concluyó que "[c]iertamente había en la Argentina la necesidad de una acción pedagógica dirigida a la despolitización de las Ciencias Sociales."4

El informe concluía que "la posibilidad de asociar el Centro, es decir, una institución latinoamericana, situada en la región, con proyectos de investigación originados y conducidos por agencias y académicos norteamericanos facilitaría en gran medida la aceptación de tales actividades de investigación por los latinoamericanos, obstaculizando la estereotipia, las distorsiones, y los malentendidos habituales sobre iniciativas de naturaleza puramente científica." También se expresaba la esperanza de que "ideologías en conflicto, grupos 
de presión, prejuicios ingenuos...que pueden [interferir] e interfieren con los procedimientos racionales necesarios...para mayores niveles en la enseñanza y la investigación" fueran superados por una institución local perteneciente al área. "Su papel intermediario podría...contribuir a superar las opiniones e 'imágenes públicas' negativas y polarizadas que a menudo oscurecen los contactos directos entre instituciones científicas latinoamericanas y agencias extranjeras.”5

Las citas del informe de la Fundación Rockefeller ilustran algunas de las preocupaciones centrales de la diplomacia cultural de EE.UU. durante la Guerra Fría. Después de 1945, sus programas culturales orientados al exterior abandonaron el acercamiento idealista defendido por los pioneros filantrópicos y científicos de la preguerra. Bajo la dirección de una élite interconectada y coordinada por el Departamento de Estado, la diplomacia cultural se tornó en un "desarrollo orgánico" de la política exterior de EE.UU. ${ }^{6}$ En América latina, EE.UU. necesitaba realzar su credibilidad para contrapesar varios factores, promesas incumplidas sobre desarrollo en la postguerra, políticas económicas impopulares, apoyo a dictadores, incidentes raciales internos, que desacreditaban las afirmaciones democráticas de su política exterior durante los años 1950s. EE.UU. necesitaba demostrar con los nuevos programas que "lo que América dice es lo que América hace."”

Los planificadores científicos de la diplomacia cultural de EE.UU. exportaron, de hecho, más que paradigmas científicos y artísticos: ellos promovieron el sueño norteamericano. La exportación de complejos sistemas de imágenes, símbolos, valores, tecnología, ciencia, y arte era al mismo tiempo la exportación de una ideología. En palabras de Emily Rosenberg, "el desarrollismo liberal combinó principios liberales con la experiencia histórica del propio desarrollo norteamericano, elevando la creencia y las experiencias de un tiempo histórico único y circunstancial de América en leyes de desarrollo pensadas como aplicables en cualquier lado." Esta ideología mantenía que "otras naciones podrían y deberían repetir la propia experiencia de desarrollo de América." La creencia secular en la capacidad de EE.UU. para "perfeccionar, aplicar leyes de mejora progresiva, y elevar a quienes estaban más abajo en la escala evolutiva" era también una fe religiosa en la misión de "América."”

A través de este período, la diplomacia cultural norteamericana complementó la política exterior tradicional con el trabajo de nuevas agencias oficiales-USIA, AID_organizaciones profesionales-SSRC NAFSA — grandes fundaciones filantrópicas-Ford, Rockefeller-y universidades públicas y privadas. Al principio, las campañas apuntaron tanto a élites como al público masivo extranjero con el objetivo de "vender Americanismo al mundo." Sin embargo, poco después las políticas principales privilegiaron la meta de influenciar las ideas de las élites culturales y políticas, ganar sus corazones y mentes, que eran quienes, a su vez, 
formarían los valores y las ideas sobre EE.UU. en sus respectivas sociedades. Diversos programas tales como el de Fulbright para el intercambio de estudiantes, o los de USIA para líderes, y de Pueblo a pueblo difundieron por todo el mundo transferencia de conocimientos técnicos y científicos, patrocinio de ediciones de libros a precios populares, y películas de Hollywood, pero también campañas de saturación que hicieron publicidad de programas de TV, de turismo en los EE.UU., o del estudio de inglés como segunda lengua.

Los estudios sobre la recepción de esas iniciativas por sociedades extranjeras son más escasos que los análisis de las políticas emisoras, y generalmente descuentan el éxito de las iniciativas de EE.UU. sin ninguna problematización. ${ }^{9}$ Sin embargo, como este ensayo demostrará, también ocurrió un proceso más complejo de negociación, resistencia, apropiación y adaptación cuando los funcionarios norteamericanos llegaban a cada país extranjero, interactuaban dentro del ambiente social y político local, y quedaban involucrados - a veces involuntariamente, a veces inevitablemente - en la dinámica del campo académico local. En otras palabras, es necesario desafiar las presunciones etnocéntricas e interesadas de una gran mayoría de diplomáticos de EE.UU. que sostenían que "existen... ciertos patrones dominantes de la sociedad industrial occidental que no pueden ser rechazados por nadie que acepte las máquinas del Oeste." 10

Para encontrar el sentido de estos intercambios interculturales, es necesario revisar las premisas que orientaron tradicionalmente a los historiadores diplomáticos. Los estudios más recientes sobre relaciones exteriores de EE.UU. han comenzado a centrarse en sus dimensiones culturales, incluyendo en su análisis información valiosa sobre las presunciones, representaciones, y símbolos que orientaron al personal y a las instituciones de la diplomacia norteamericana en el nivel más o menos consciente, pero que, sin embargo, habían quedado inadvertidas por acercamientos anteriores. ${ }^{11}$ Así, el acercamiento cultural acierta al ir más allá de los acercamientos interpretativos del tradicionalismo y del revisionismo incluyendo temas que van más allá de estrategia y economía política. Además, el énfasis de este acercamiento en la investigación multiarchivística para reconstruir, criticar, e interpretar el proceso en cada nivel analítico coincide con el creciente interés de los investigadores de EE.UU. en la consideración de las perspectivas del lado receptor de su diplomacia a través del estudio de archivos extranjeros.

La nueva ola de estudios sobre la globalización también ha acentuado la necesidad de escuchar ambos lados de las transmisiones culturales. ${ }^{12}$ Las tentativas de entender la expansión global de instituciones de la nación más poderosa deben evitar máximas como la de Weber, cuando dice que "sólo en la civilización occidental han aparecido fenómenos culturales que (como nos gusta pensar) se asientan en una línea de desarrollo que tiene significación y valor universales."13 
Siguiendo a Roberto Russell, se puede decir que "la formación de una sociedad global no debe implicar apenas terminar con la diversidad a través del triunfo de un idea" sino que, de una manera normativa, ésta debe ser concebida como un proceso que combine la idea de un lugar, la Tierra, mientras que valora la diversidad positivamente. ${ }^{14}$

Los estudios sobre globalización han seleccionado a las instituciones culturales más visibles del imperio americano- los conceptos de " modo de vida norteamericano," “Americanización, “y " anti-Americanismo"-para considerar la relación entre fuerzas norteamericanas y locales en todo el mundo. Sin embargo, esos estudios deben entender los procesos de integración global y fragmentación local como dinámicos e interactivos, evitando la reproducción del marco analítico unidimensional del proceso de globalización económica. ${ }^{15}$ La presunción de que la globalización cultural consiste en una confrontación entre dos lados, al parecer, distintos, que se encuentran en un vacío no sólo yerra al ignorar la densidad histórica de contactos anteriores en el lugar bajo estudio sino que también descuida la presencia posible de dos o más fuerzas extranjeras superpuestas - es decir, de representantes de diversos imperios. Los estudios sobre post-colonialismo constituyen una fuente provechosa de estudios de caso para esto, ya que trabajan generalmente en áreas de influencia imperial superpuesta a través del tiempo. ${ }^{16}$

Además, la discusión sobre la expansión de las instituciones de EE.UU. más allá de su frontera nacional tiene que considerar la influencia de la creencia en el "excepcionalismo" norteamericano. El celo para destacar las características particulares de la consolidación de la formación social de EE.UU. ciertamente ha influenciado a varias generaciones de académicos norteamericanos. Sin embargo, este acercamiento parece haber confundido las condiciones históricas singulares de ese desarrollo con factores esencialmente diferentes de una civilización que se quiere interpretar como única. Asimismo, el impacto de la Guerra Fría en la política nacional de EE.UU. impuso severos límites ideológicos y políticos sobre el desarrollo de las instituciones democráticas locales, tales como el McCarthyismo durante y después del período del senador Joe McCarthy, que ejerció influencia efectiva sobre las instituciones educativas del país. Ambos factores ayudaron a la consolidación de una interpretación nacionalista del comportamiento de EE.UU. dentro del sistema internacional que puso con frecuencia a la academia norteamericana en desacuerdo con estudios más inclusivos y ambiciosos de otras partes del mundo, es decir, originados lejos de los lugares de expansión de EE.UU. El concepto de imperio ${ }^{17}$ también ha desequilibrado históricamente a los académicos realistas, que tendieron a explicar la expansión de EE.UU. con interpretaciones subjetivas tales como "idealismo moral," "histeria total," o "desvío de menor importancia en la marcha de la historia americana," en contra de los académicos 
revisionistas, que tendieron a explicar esa expansión como el producto de intereses económicos, ampliamente interpretados.

La complementación de análisis estructurales de sociedad y poder con un énfasis postmoderno en identidad y contingencia intenta ir más allá de esta oposición. El interés en la naturaleza y la construcción del poder de EE.UU., así como su desarrollo y puesta en práctica, han ampliado las fuentes de evidencia consideradas anteriormente para centrarse también en las prácticas y representaciones que acompañaron la presencia de EE.UU. en el exterior. Por otra parte, también se ha orientado sabiamente el estudio del imperialismo de EE.UU. al examen de sus efectos sobre el campo interno, es decir, sobre la constitución de la sociedad, cultura, e ideología de EE.UU. ${ }^{18}$

De todos modos, el acercamiento cultural está lejos de ser una tendencia homogénea. Hay interés en la exploración de la intersección de cultura y poder, y en la construcción social de la vida política a través de la incorporación de nuevas categorías de análisis que enriquecen el estudio de la realidad, tales como la influencia de género, etnia, clase, o lengua en las presunciones de agentes e instituciones. Sin embargo, varios estudios han sido influenciados pesadamente por interpretaciones radicales del post-estructuralismo a través de la presunción de que la realidad es un producto de prácticas lingüísticas y textuales. Esta postura antipositivista y anti-idealista sostiene que la lengua es auto-referencial, considerando discurso y estrategias discursivas como las posturas supremas que definen la realidad o que llevan temas y objetos a la existencia. Su esfuerzo principal se centró en minar conceptos dominantes, tales como "Estado," "nación," o "soberanía," con cuidadosas genealogías que buscan "privar a estas formaciones sociales [y a los conceptos] del aura de un designio natural o de un orden moral intrínseco que tiende a formarse alrededor de ellos." 19

Aunque este acercamiento puede ser provechoso como herramienta crítica, también debilita la tentativa de estudiar la cultura más allá del nivel de lengua y el discurso. El estudio de las formas simbólicas de dominación tiene que complementar, y no excluir, las fuentes de evidencia más amplias que la historia diplomática más moderna está dispuesta a considerar. La ventaja de analizar críticamente las múltiples implicancias de los medios simbólicos de la expresión y de la representación no debe evitar que las integremos en un acercamiento más ambicioso que también valore la esfera cultural como parte influyente del procedimiento de toma de decisiones, aunque no como única herramienta. Debe pensarse, en cambio, que el sistema de prácticas, instituciones simbólicas, $\mathrm{y}$ significados insertos en el comportamiento diario de un grupo social tiene ciertamente relación con la estructura social que, al mismo tiempo, las forma y es formada por ellas. La cultura es un componente integral del poder, al mismo 
tiempo "socialmente constituida" (es un producto de la actividad del presente y del pasado) y "constituyente social" (es una parte del contexto significativo en el cual la actividad ocurre). ${ }^{20}$ Las culturas "son producidas en relación con ellas mismas, en una dialéctica de compromiso que tiene lugar en contextos de poder desigual y que implica préstamos, expropiaciones y transformaciones recíprocos." ${ }^{21} \mathrm{La}$ recuperación de conceptos de dinámica social y su aplicación al análisis de la política exterior en general - diseño, desarrollo, recepción, y nueva valoraciónpuede proporcionar hallazgos valiosos sobre la naturaleza multiforme del poder en las relaciones internacionales.

El esfuerzo para explorar acercamientos heterogéneos y complementarios, "descentrar análisis, desagregar reificaciones, y restaurar la agencia en la narrativa histórica" hasta el momento parece haber obtenido buenos resultados. ${ }^{22}$ Por ejemplo, nuevos estudios sobre relaciones exteriores entre EE.UU. y América Latina se centran en las "zonas de contacto" del imperio para analizar el "encuentro" con el Sur. Estos nuevos conceptos sugieren un esfuerzo genuino para integrar paradigmas modernos y postmodernos, y así proporcionar una estrategia útil para entender los elementos múltiples - materiales y discursivos — que participan en ese proceso, así como la naturaleza interactiva y dialéctica de esos contactos. ${ }^{23} \mathrm{El}$ término "zona de contacto" procura ampliar la presunción sobre el número y tipo de casos en los que ambas regiones "se encontraron." EE.UU. ejerció su influencia en América Latina-y viceversa — en muchas áreas más allá de la diplomacia, tales como comercio, contrabando, migraciones, religión, deportes, academia, etc. El término "encuentro" enfatiza el hecho de que esas interacciones, "generalmente marcadas por desigualdad y conflicto, si no coerción, pero también con posibilidades interactivas, improvisadas...no debería ser vista en términos de separación...sino en términos de co-presencia, y de prácticas y presunciones interconectadas." ${ }^{24}$

Algunos de los conceptos discutidos han sido provechosos para este estudio de caso. En primer lugar, en lo que concierne a la globalización de la ciencia, se observa que la expansión imperial de la academia norteamericana sobre el campo cultural de Argentina, lejos de encontrar un espacio vacío, entró a una tradición rica y activa de diversas escuelas científicas y filosóficas europeas que la precedieron por décadas. Además, los programas académicos de EE.UU. no fueron apenas canales unidireccionales para la circulación de paradigmas; por el contrario, el campo de las Ciencias Sociales de la Argentina fue un sitio de encuentro en donde personas, instituciones, ideas, y valores de ambos países interactuaron dentro de un marco de relaciones asimétricas de poder. Este ensayo se centra en los contactos entre agentes diplomáticos y académicos de EE.UU. y de Argentina para examinar la dinámica del encuentro y su influencia sobre la relación bilateral.

¿Cómo fue recibida la diplomacia cultural norteamericana por las élites 
culturales argentinas? ¿Cuál fue el impacto de esas iniciativas en la dinámica del campo cultural local? A través del período del "desarrollismo," las discusiones entre los intelectuales argentinos fueron influenciadas por la fuerte herencia del Peronismo, distintas corrientes marxistas, y un vago discurso nacionalista que recorrió todo el espectro político. ${ }^{25}$ En otro artículo he demostrado que los programas e instituciones organizados y financiados por EE. UU. dividieron profundamente el campo académico local en la forma que sugiere el sociólogo francés Pierre Bourdieu. El recientemente creado sector de nuevos intelectuales rápidamente se convirtió en una nueva élite dentro del campo académico local a través de su disposición-su asociación al "más moderno" campo cultural norteamericano y su conexión directa con ese campo académico de referencia-así como por su posición - condiciones de trabajo mejores, más estables y más ricas en recursos. La naturaleza transnacional de estos "sitios de contacto" tuvo consecuencias múltiples, imprevistas y, a veces, contraproducentes para su recepción y desarrollo locales. ${ }^{26}$

La politización inmediata de las diferencias disciplinarias dentro del campo académico argentino, y las acusaciones de colaboración con EE.UU. no podían sorprender en un campo cultural que tenía tan poca autonomía de la política nacional. El caso particular de la expansión de las Ciencias Sociales de orientación norteamericana en la Argentina a finales de la década de 1950 reprodujo patrones típicos de la Guerra Fría. Las discusiones permanentes sobre la evolución de las relaciones culturales bilaterales, la sospecha generalizada de colaboración con actividades de inteligencia de EE.UU., y la desconfianza lisa y llana de la simple motivación científica, académica, filantrópica, o humanitaria de las iniciativas, crearon obstáculos y resistencias. Por otra parte, algunos casos justificaron completamente esa suspicacia, como el escándalo alrededor del Proyecto Camelot de American University, en 1965. En esa ocasión, el descubrimiento de que los datos recogidos sobre marginalidad social en América Latina serían aplicados a estudios estratégicos de contrainteligencia por el Departamento de Defensa de EE.UU. constituyó un duro golpe al prestigio de la diplomacia cultural norteamericana. Como una consecuencia directa de ello, poco tiempo después, una manifestación agresiva de los estudiantes de la Universidad de Buenos Aires durante la visita del prestigioso huésped Walt W. Rostow provocó la dimisión del Rector de la institución y la interrupción de iniciativas similares. ${ }^{27}$

Un ejemplo de las violentas críticas de la izquierda local contra el imperialismo cultural norteamericano puede verse en un artículo que mantenía que “...debido al hecho de que el imperialismo es la etapa de la descomposición, el deterioro de los términos de intercambio también afecta al intercambio cultural, el cual se convierte en una invasión que sofoca las culturas nacionales." El artículo 
agregaba que "los valores morales de la investigación científica y del bienestar humano fueron substituidos por los intereses de monopolios, y los intelectuales desesperados descubrieron que los suyos no coincidían con los de su patria sino con los del opresor." ${ }^{28}$ Otro ejemplo de la posición crítica es la respuesta a un artículo de William Benton, uno de los líderes en el desarrollo de la diplomacia cultural de EE.UU., sobre las ventajas de universidades patrocinadas por EE.UU. en América Latina, que supuestamente "darían un significado humano a la revolución tecnológica en curso." Esa clase de universidad "apolítica y no sectaria," indicaba la crítica, tornaría a los estudiantes impotentes frente al directorio corporativo. Por otra parte, la enseñanza sería en inglés, y promovería a los estudiantes a EE.UU., tornando al sistema en un apéndice de la educación norteamericana [con el propósito de] crear una casta local capaz de manejar la conquista norteamericana del continente." 29

Un informe de la Fundación Ford que evaluaba los logros de la institución en Argentina era particularmente ilustrativo de la percepción norteamericana de la academia local durante ese período. El informe decía que "el problema en las universidades es más que financiero...entre los estudiantes y los profesores más jóvenes, una ola de corrientes ideológicas - Nacionalismo y Populismo, Peronismo y anti-imperialismo, algo de anti-intelectualismo y un arsenal de variantes del Marxismo - se combinan para minar el proceso académico básico y prevenir cualquier discurso lúcido." En particular, el informe destacaba la emergencia reciente de una corriente contraria a lo que se definía como "imperialismo cultural... [por el cual] algunos estudiantes rechazan a cualquier profesor que tenga un grado doctoral conseguido en el extranjero... [o] aún más notablemente, que rechazan leer a Marx considerando que es un extranjero y, por lo tanto, no relevante para la Argentina." 30

Más allá de estas apreciaciones sobre el sistema de educación superior local, evidentemente el informe no consiguió ir más allá de la descripción de obstáculos puramente locales, y hacia un análisis de las causas de la situación. El trabajo de la Fundación se limitaba a la descripción mecánica del sistema local y a una evaluación de sus diferencias con el sistema de EE.UU., con poca evidencia de haberse beneficiado de información adicional de la Embajada de EE.UU. Los análisis más incisivos de los funcionarios políticos podrían haber permitido una comprensión más profunda, que relacionara los problemas de la universidad argentina con la difícil transición política del país. En cambio, la identificación ciega de los funcionarios de la Fundación con el paradigma de la universidad norteamericana y con la "modernización"- así como con la misión nacional de trasplantarlos al exterior-limitó su percepción del campo local.

Otro hecho frecuente en el sitio de contacto era la típica obsesión de la 
Guerra Fría de EE.UU. con la seguridad local, lo cual también afectó la expansión académica transnacional. En un caso característico, la Fundación Rockefeller recibió críticas en EE.UU. exigiendo que no se perdiera "ningún dinero norteamericano en gente con inclinaciones marxistas," como el sociólogo Gino Germani, el prestigioso italiano que había fundado la Sociología moderna en la Argentina a través de un acuerdo entre la Fundación y la Universidad de Buenos Aires en 1958. Tratando su caso, un académico norteamericano opinaba que "el hecho de que la afiliación política hiciera cualquier diferencia en un hombre de ese tipo era un asunto debatible." Y, en un intento por formular un criterio por el cual científicos locales pudieran ser excluidos de los planes de la Fundación, concluía que "'alguien que puede corromper a la comunidad local' [era] un buen comienzo." Sin embargo, los funcionarios solamente se tranquilizaron cuando supieron que Germani había recibido previamente varias visas norteamericanas, y que había adquirido prestigio y respeto durante su estancia en Berkeley y Chicago, lo cual era considerado como "señales seguras." 31

En otro caso, la Fundación Rockefeller consideraba una beca para el prestigioso historiador José Luis Romero, "el único académico del área de investigación sobre historia interpretativa contemporánea con el cual la Fundación podría trabajar." 32 Sin embargo, antes de designar a Romero la Fundación tuvo que pedirle que se identificara porque no habían encontrado su nombre en la popular guía norteamericana Who's Who? ni en los listados de más de treinta organizaciones prohibidas en EE.UU., incluyendo el Indice cumulativo a las publicaciones del Comité sobre actividades antiamericanas, el Indice compuesto del Comité McCarthy, y el Comité McCarran sobre extranjeros en EE.UU. ${ }^{33}$

Mientras que en el caso de Germani, los funcionarios observaban que éste probablemente estaba "a la izquierda del centro," también destacaban que ambos hombres "recibían ataques del catolicismo argentino," "de colegas pasados de moda," y de grupos de izquierda que los acusaban de ser agentes imperialistas debido a su acercamiento a la academia norteamericana. ${ }^{34}$ Ambos casos indican que estos programas en el campo de la ciencia local, apoyados por agencias políticas de EE.UU., eran de tal incidencia-y su acoplamiento al país hegemónico en el continente tan visible - que proporcionaban un blanco fácil para ataques de los grupos ideológicos más diversos.

El encuentro transnacional también ocasionó episodios interesantes de resistencia y negociación, como la reacción al término "modernización," que era la palabra clave para el nuevo acercamiento paradigmático de las ciencias sociales norteamericanas. Las reacciones contra esa idea-fuerza eran múltiples y variadas. Por ejemplo, una publicación católica liberal resaltaba que su generación había descubierto "un nuevo mineral: el oro de la inteligencia," que había alimentado la 
revolución científica y el proceso de modernización. Sin embargo, también advertía contra la "dominación del productivismo" que había invadido la organización de la ciencia y que había afectado el compromiso primario de ésta con el bienestar humano. ${ }^{35}$ En un intento similar, pero desde acercamientos diferentes, dos científicos de rica formación bicultural, como Torcuato Di Tella y Kalman Silvert, describían con profundidad los problemas de la transición latinoamericana-la trayectoria a la modernización - en 1961. Di Tella se centraba en la carencia de una "intelligentsia" como grupo social, lo cual explicaba tanto el sentido de aislamiento histórico del intelectual latinoamericano como su necesidad de asociarse a otros grupos de presión - corporaciones, militares, sindicatos, gobiernos-que finalmente lo subordinaban, reproduciendo así la debilidad del campo cultural; esa carencia de autonomía evitaba que el grupo cobrara fuerza y legitimidad dentro de su sociedad. Por su parte, Silvert se centraba en el proceso de modernización en América latina, que había impulsado a la gente a un "salto desde la lealtad a la familia...a identidades trascendentales... sin un nivel intermedio de lealtad impersonal a la comunidad." El resultado político de esa aceleración había sido un "Estado nacional considerado como anti-cristiano, de nacimiento depravado y bajo el pecado original, o como un dios que debía ser adorado en sí mismo." La transición acelerada había obstaculizado la creación y reproducción de un grupo autónomo de intelectuales que actuaran al mismo tiempo como pensadores y guardianes de su propia sociedad. ${ }^{36}$

La aplicación de subsidios extranjeros para sustentar esa "modernización" también levantaba protestas, como por ejemplo las del Consejo Superior de la Universidad de Buenos Aires, el órgano de gobierno de la Universidad, integrado por representantes de graduados, profesores, y estudiantes. Un estudiante graduado expresaba el pensamiento generalizado sobre el origen de esos subsidios, los cuales "generalmente implicaban la existencia de condiciones implícitas sin las cuales no serían otorgados." Desde esta posición, los subsidios estaban viciados desde su origen por su "relación inmediata con el interés de los grupos pequeños, pero con gran poder, que se oponían al interés general, popular.” Además, era necesario decidir si se quería "una universidad que sirviera al desarrollo nacional independiente, o 'apéndices' que desplazarían la enseñanza y la investigación del país verdadero al refinamiento de los laboratorios." ${ }^{37}$

¿Qué sucedió en el Instituto Di Tella como sitio de contacto entre los programas de Ciencias Sociales patrocinados por EE.UU.y los centros de investigación locales? En el caso de la Sociología, los ataques que tenían como objetivo la conexión con la ciencia norteamericana eran demasiado obvios, puesto que la misma Fundación Ford había financiado el primer programa en la Universidad de Buenos Aires, en 1959. ${ }^{38}$ Además, la supuesta neutralidad ideológica de esa Sociología era puesta 
en duda. Por un lado, Gino Germani, su representante principal, era acusado por católicos de "ser judío, disponer a los estudiantes contra el servicio militar, y poner en duda la esencialidad de la familia como unidad social humana básica, aparte de varias acusaciones de inadecuada conducta sexual." ${ }^{39}$ Por otra parte, los marxistas razonaban que " ¿si se acepta que la observación de la realidad está mediada por las categorías subyacentes y por las operaciones conceptuales que la hicieron posible, cómo se podía continuar ocultando las decisiones implícitas detrás de la Sociología norteamericana? ... Cómo [era] posible tratar de comprender las condiciones dramáticas del subdesarrollo basándose en el paradigma que había mantenido a América Latina en esa condición?" ${ }^{40}$ Entre los sociólogos norteamericanos, solamente C. Wright Mills era elogiado por su "rebelión contra las prácticas antidemocráticas y militaristas del capitalismo contemporáneo," y por su crítica a la futilidad y la falta de generosidad de la Sociología norteamericana, especialmente su empirismo abstracto." ${ }^{41}$

Las observaciones sobre aquellas actitudes "más modernas" hacia la ciencia en aquella frontera de hibridación intercultural también podían aparecer en temas simples. En una ocasión, mientras que el informe de un funcionario de la Fundación Ford sobre la calidad y funcionamiento del Centro de investigaciones económicas del Instituto Di Tella era positivo, también contenía quejas por el lento progreso de los proyectos de investigación. El informe apuntaba al "patrón psicológico local 'mañana-siesta," y resaltaba que "ese rasgo [era] perceptiblemente menor entre los que habían estudiado en el exterior," identificando esa experiencia con una actitud más comprometida con la productividad. Además, una discusión entre profesores locales sobre un nuevo tipo de Dirección rotativa para el Centro parecía confirmar esa tendencia hacia la mutación en las opiniones estereotípicas. Sin embargo, en contradicción con esa tendencia aparente, fue el propio funcionario de Ford quien se alarmó ante esa elección porque, en su opinión, esa decisión daría lugar a una dirección débil, en contra del deseo de la Fundación de mantener una personalidad fuerte durante un largo periodo como única forma de corregir algunas debilidades locales. En contraste, los investigadores argentinos-la mayoría de los cuales había tenido experiencia en el exterior-privilegiaban "la investigación como la actividad más importante, y no la dirección de estilo Criollo - con un contenido fuerte de caudillismo - que era incompatible con las necesidades de la ciencia moderna." Esta posición puede ser considerada como la emergencia consciente de la voluntad local de modificar dinámicas tradicionales de las instituciones locales. ${ }^{42}$ Sin embargo, es interesante observar que, mientras que ambas partes intentaban fortalecer el desarrollo de la nueva institución local, fueron los argentinos — quienes se sentían más responsables por el establecimiento de esa nueva clase de institución en el campo local—quienes parecen haberse 
inclinado más decididamente y con mayor iniciativa hacia "la forma moderna" a la cual adaptarse. Este rasgo también puede apreciarse en la discusión de los planes para un nuevo centro en la cual los científicos argentinos reclamaban más apoyo de personal extranjero, admitiendo que la sola presencia de argentinos con el grado de Ph.D. no sería suficiente para proveer al personal y a los estudiantes la dirección fuerte y estable que éstos necesitarían: "Usted sabe, con el complejo colonial que tenemos..." "43

Más allá de estas percepciones, la aplicación de la tradición científica fue, quizás, el tema substancial de muchas discusiones, y el que bloquearía eventualmente el acceso de ciertos programas académicos a la Argentina. Consultado sobre un plan propuesto para un Centro de psicología industrial en el Consejo Nacional para Investigación Científica y Tecnológica (CONICyT), un prestigioso académico local advertía contra la especialización excesiva dentro de un campo específico, pues eso sería contrario a las necesidades del país. En una referencia obvia al acercamiento positivista de la tradición norteamericana, y en comparación con acercamientos más amplios de otras escuelas europeas, observaba que "la tendencia que considera que la Ciencia debe inventar modelos y después considerar si la Naturaleza coincide con esos modelos siempre me ha resultado extraña." Por otra parte, "en un ambiente que confía tanto en la teoría como el nuestro, un laboratorio de esa clase puede dañar desarrollos posteriores en ciencia experimental." ${ }^{44}$

Lainfluencia delasdistintastradicionescientíficas en losproyectosacadémicos conjuntos entre la Fundación Rockefeller y el Instituto Di Tella fue discutida cuidadosamente en las negociaciones. La resistencia a paradigmas dominantes en EE.UU. y la estrategia de una cuidadosa aproximación crítica caracterizó a los campos de la Sociología, la Psicología, y la Historia. Los economistas argentinos del Instituto Di Tella, sin embargo, estuvieron más dispuestos a tender un puente entre esas tradiciones. Aunque rechazaban el supuesto "valor neutro" de las recetas norteamericanas para el desarrollo, ellos también entendían claramente su doble necesidad de desafiar a esa tradición científica y de conseguir nuevas herramientas para el desarrollo local. Los economistas decidieron concentrarse, entonces, en la aplicación antes que en la teoría-y después en la "teoría aplicada"-porque necesitaban demostrar que el trabajo empírico combinado con las herramientas modernas de análisis sería provechoso para el país." ${ }^{45}$ Sin embargo, esa nueva camada de economistas argentinos también estaba dispuesta a confrontar con los supuestos prácticos y teóricos de la emergente escuela de la dependencia de América latina. En 1966, por ejemplo, negaban que "la poca correspondencia entre la teoría americana y la realidad latinoamericana" tornara a los estudiantes con grados académicos extranjeros inútiles para las economías subdesarrolladas.'Existen principios económicos fundamentales ... que deben ser estudiados a fondo, 
culminando con el entrenamiento para idear modelos y probarlos a través de estadistics [sic]." E incluso si esa diferencia existiera, agregaban, la gran necesidad de graduados de alto nivel de la Argentina la obligaría a enviarlos a estudiar en EE.UU. de todos modos." Además, observaban que un número cada vez mayor de universidades de EE.UU. incorporaba cursos sobre países subdesarrollados. El informe concluía que la Argentina estaba en un punto de desarrollo en el cual una escuela local para estudiantes de post-grado podría ayudar a ahorrar el tiempo y dinero invertidos en su salida al exterior y en su posterior readaptación al país." ${ }^{\prime 4}$

El Consejo de Dirección del Instituto Di Tella tomó una posición más cautelosa con respecto a la naturaleza de su relación con las ciencias sociales norteamericanas. El Director Ejecutivo, Enrique Oteiza, advertía durante uno de sus discursos semanales en la radio contra el reduccionismo capitalista y los acercamientos socialistas, llamándolos "dos clases de recetas que no respondían a la realidad." Ambas estrategias de desarrollo habían traído fracaso y frustración a naciones más pobres al trasplantar copias indistintas de países desarrollados, ya que el ingrediente más importante - es decir, la capacidad de crear y de absorber cambios continuos - era invisible y no factible de ser "copiado." ${ }^{\prime 7}$ En forma similar, en una de las reuniones entre los funcionarios de la Fundación y los académicos locales, dos de éstos lanzaron advertencias en contra de algunos aspectos de una oferta para investigar el crecimiento y la estructura de la población en América Latina. "En esa propuesta," insistían, "[hay] teorías de desarrollo económico implícitas. Y advertían que "[existe] el peligro de que una teoría de desarrollo económico sea aceptada implícitamente como la teoría, sin la prueba crítica necesaria." 48

Para Torcuato Di Tella, Jr.,el Instituto Di Tella reconocía la ventaja de que la nueva generación de "científicos sociales argentinos hablara la misma lengua" que los científicos norteamericanos. Sin embargo, el investigador también recordaba que él "había decidido diversificar la formación de los graduados que iban al exterior para evitar la posibilidad de que, a su regreso, se convirtieran en una 'sucursal de la Universidad de Chicago' en el país... Intentamos enviarlos a diferentes universidades en EE.UU. y Europa. Lo importante era que, a su regreso al país, tuvieran que interactuar en el medio local para nacionalizar su entrenamiento extranjero y reprocesar esas tradiciones académicas distintas." Con énfasis especial, Di Tella señalaba a los acuerdos que se habían constituido en modelo de la época, tales como aquél entre Universidad Católica de Chile, Universidad de Chicago, y Fundación Ford, como aquello que debía ser evitado. Di Tella remarcaba que "siendo esos economistas los únicos altamente entrenados en el país, no habían encontrado ningún desafío serio a su acercamiento conservador y tecnócrata, y finalmente se habían constituido en la base hegemónica para el experimento de Pinochet." 49 Siguiendo esa línea de pensamiento, otros investigadores argumentaban que "la 
hegemonía actual de la tendencia tecnócrata y unidisciplinaria que se preocupa solamente por los modelos macroeconómicos financieros" probó que los nuevos economistas eran "los menos críticos de la ciencia norteamericana, y que no habían podido para crear su propia escuela. ${ } 50$

El complejo proceso de circulación de valores, representaciones, y símbolos en el sitio de encuentro entre dos tradiciones científicas nacionales puede ser apreciado con más profundidad observando al personal de los programas de EE.UU. De hecho, después de más de una década en el sitio de encuentro, la experiencia había transformado a los mismos funcionarios de la Fundación: el tiempo y la experiencia habían reformado sus presunciones sobre la dinámica de la exportación de programas y paradigmas de Ciencias Sociales. Sin embargo, su reconocimiento de las limitaciones de su iniciativa de modernización y reemplazo paradigmático, y de la necesidad de un diálogo constante entre su misión nacional y las condiciones particulares del campo local-lo profesional, lo político, lo ideológico - generalmente llegó demasiado tarde.

Por ejemplo, en sus observaciones sobre el brillante trabajo teórico del Centro de investigaciones económicas del Instituto Di Tella, un funcionario de la Fundación Ford reconocía que, contrariamente al consejo de la Fundación, "el modelo del Centro había sido derivado de una crítica a la teoría económica convencional para desarrollar modelos teóricos correspondientes a las presunciones institucionales sobre funcionamiento de economías dependientes. ${ }^{51}$ En un nivel similar, ese funcionario de Ford recibió una respuesta probablemente iluminadora después de su informe desfavorable sobre el Centro de investigaciones sobre educación del Instituto Di Tella. De hecho, el investigador argentino cuyo trabajo había sido criticado indicaba que "ese informe le había recordado aquéllos de veinte años antes, hechos por extranjeros que solamente pensaban en términos de modelos europeos o norteamericanos, con énfasis en teoría y modelos causales." Aquellos acercamientos "reflejaban ignorancia sobre los apremios locales y "producían estudios diagnósticos, descriptivos...nada sofisticados," que demostraban "gran inexperiencia en America Latina." Como consecuencia, el funcionario norteamericano y su supervisor en Nueva York terminaron "preguntándose si alguna vez ellos mismos habían examinado sistemáticamente los estándares por los cuales juzgaron sus propios programas en Ciencias Sociales, particularmente con respecto a su importancia y viabilidad en America Latina." ${ }^{52}$ Para 1971, un experimentado funcionario de Ford concluía que "la selección de disciplinas en la Argentina, en cierta medida, [había respondido] al clima del país, [y sido] condicionada por la realidad local, "pero también reflejaba nuestras propias presunciones y preconcepciones" sobre el proceso de desarrollo y sobre las variables básicas que determinan el avance de un país hacia la modernización." 53 
Durante una entrevista centrada en la transferencia de paradigmas científicos a través de fronteras culturales, Torcuato Di Tella Jr. elaboró una hipótesis estimulante sobre la evolución ideológica de los funcionarios de fundaciones norteamericanas que habían trabajado en América Latina. A través de su extensa experiencia en el Sur, indicaba, los primeros funcionarios, "esos buenos, viejos liberales... moderados y progresistas en su país...se sorprendieron por el rechazo generalizado que recibieron de los intelectuales latinoamericanos." En un esfuerzo para entender ese nuevo ambiente, "ellos mismos se hicieron cada vez más izquierdistas...compraron el mito de los grupos latinoamericanos radicalizados, y empujaron sus proyectos a través de las burocracias de las Fundaciones, terminando por ser los creadores de refugios en el exterior para intelectuales latinoamericanos exiliados, y de las campañas a favor de los derechos humanos durante los años 1970s y 1980s." 54

En otra entrevista, Oteiza confirmaba este patrón de evolución ideológica de los funcionarios jóvenes norteamericanos provenientes de los recientemente creados Programas en Estudios Latinoamericanos de las universidades de EE.UU. Esos investigadores de campo habían estado en el terreno, trabajando codo a codo con graduados argentinos de su misma generación que compartían su universo cultural norteamericano, "que hablaban el mismo lenguaje." Sin embargo, aunque Oteiza identificaba una actitud progresista de parte de estos académicos y funcionarios, también afirmaba que la diplomacia cultural norteamericana había sido ideada principalmente por una asociación entre los mundos de los negocios, la academia, las fundaciones filantrópicas, y el Gobierno: "Hay una tradición por la cual, en la diplomacia cultural, toda esa gente rota permanentemente, como en el juego de las sillas...; sin embargo, aunque la academia ejerce cierta influencia, finalmente es penetrada por las decisiones estatales. Ése es el producto del Norte que impacta en el Sur." 55

En el mismo plano, Torcuato Di Tella, Jr. prefería pensar más allá del proceso de negociación y apropiación entre los campos científicos de ambos países para centrarse en los agentes de ese intercambio. Su valioso análisis sugería que el personal norteamericano que estaba en contacto con investigadores locales, y que estaba a cargo de la negociación específica del intercambio diplomático cultural, no actuaba simplemente como brazo ejecutivo de los programas. Estando en la línea del frente, estos funcionarios también actuaban como vehículo para la circulación, el contacto, el intercambio, y los préstamos de múltiples elementos materiales y simbólicos. Cuando volvían a sus puestos en EE.UU., se convertían en algo más que vehículos de la "receta para el desarrollo de su país," para decirlo en términos de Rosenberg. De hecho, para entonces, ellos llevaban consigo no sólo algunas lecturas latinoamericanas críticas de las ciencias sociales de EE.UU. , teoría de la 
dependencia, teoría del imperialismo, crítica de CEPAL a la teoría del desarrollo, sino también una sólida opinión sobre las condiciones para el desarrollo social y político en las fronteras del área de influencia de EE.UU. Y mientras que varios de ellos estaban maduros para considerar, e incluso para aceptar, algunas de esas opiniones, otros hasta se convirtieron en partidarios activos de la función crítica que muchos de los nuevos intelectuales ocuparon en América Latina. Una implicancia de este cambio es que los estudios sobre las consecuencias internas del imperialismo de EE.UU. debería estudiar cuidadosamente el patrón de comportamiento de los funcionarios de campo después de su retorno al país. La comprensión exacta del impacto de esos "reformistas reformados" en la densa red de la diplomacia cultural norteamericana requeriría el estudio de muchos casos y carreras personales, lo cual supera la capacidad de este estudio limitado.

Sin embargo, los representantes de la diplomacia cultural norteamericana hacia la Argentina pueden ser tomados como evidencia - y testigos - de los efectos del creciente proceso de la mundialización sobre esa área de frontera. De hecho, cuando las instituciones norteamericanas se convirtieron en parte del sistema local, los funcionarios de campo norteamericanos pudieron apreciar en forma directa la dinámica de la confrontación entre diversas tradiciones científicas dentro del campo cultural criollo. Esas confrontaciones emergieron en términos de luchas de posiciones dentro del campo académico y estuvieron en relación directa con las inestables instituciones políticas del país, generando ataques cruzados a menudo expresados en términos ideológicos ásperos, pero contradictorios. Esta dinámica podría ser mejor comprendida si fuera interpretada como una confrontación provocada por el traslado de tradiciones científicas imperiales al campo cultural local: dentro de ese marco, la asociación de algunos investigadores locales con foros científicos extranjeros constituyó su poder político y simbólico dentro del débil campo cultural nacional. La poderosa intervención organizada por la cruzada cultural norteamericana de los años 1950s - inevitablemente identificada como una extensión de la política exterior de EE.UU.-_impuso un cambio no sólo en las relaciones de poder dentro de la academia local sino también en las estrategias de representación de cada tradición científica.

La iniciativa diplomática cultural norteamericana que comenzó en los años 1950s fue claramente exitosa en su intento por crear un "nuevo sujeto" en América Latina. El grupo de científicos sociales que surgió en los nuevos centros de investigación de la región, como en el caso del Instituto Torcuato Di Tella en Buenos Aires, compartió el lenguaje científico y técnico de las iniciativas norteamericanas y colaboró en la expansión económica que consolidó la influencia de EE.UU. Las ciencias sociales latinoamericanas fueron beneficiadas por iniciativas que permitieron el desarrollo más estable de actividades que, de otra manera, solamente 
eran posibles en los centros de poder internacional. Sin embargo, el contacto entre las dos naciones, o más precisamente, entre sus tradiciones, instituciones, e ideologías científicas, las cuales se encontraron en el campo cultural de la Argentina, e interactuaron dentro del sistema de poder local, abrió un rico proceso de intercambio cultural que excedió los objetivos de la política exterior de EE.UU. Los sitios de contacto de la expansión cultural norteamericana constituyen un terreno fértil no sólo para el estudio de las políticas imperiales sino también de los múltiples agentes, instituciones, e ideas, nacionales y transnacionales, que se encontraron mutuamente en ese proceso.

\section{NOTAS}

I "International Center for Comparative Social Research (un proyecto especialmente dedicado a la investigación en sociedades de América Latina)" I. Folder 86 "Instituto Di Tella," Box 10, Series 30 I S, Record Group I.2, Rockefeller Archive Center, North Tarrytown, New York [desde ahora RAC].

2 "International Center for Comparative...," 2.

3 "International Center for Comparative...," 4.

4 "International Center for Comparative...," 4-5.

5 "International Center for Comparative...," 6.

6 La historia de la diplomacia cultural de EE.UU. está desarrollada en la disertación doctoral del autor, "Selling Americanism Abroad: American Cultural Diplomacy toward Argentina, 1953-1963," SUNY at Stony Brook, History Department, January 1999. Otros trabajos e interpretaciones son Frank Ninkovich, The Diplomacy of Ideas (Cambridge, 1981); Mark T. Berger, Under Northern Eyes: Latin American Studies and U. S. Hegemony in the Americas, $1898-1990$ (Bloomington, 1995); Fitzhugh Green, American Propaganda Abroad (New York, 1988); Emily Rosenberg, Spreading the American Dream (New York, 198I); José Manuel Espinosa, Inter-American Beginnings of U. S. Cultural Diplomacy (Washington, D.C., 1976); Kenneth L. Kornher, The Psychological Instrument of U.S. Foreign Policy: USIA, 1953-1963 (Ann Arbor, 1970); Charles Frankel, The Neglected Aspect of Foreign Affairs (Washington, D.C., 1965); Charles Thomson and Walter H. Leaves, Cultural Relations and U. S. Foreign Policy (Bloomington, 1963).

7 PMS 30-58-7 "The Credibility of What America Says Abroad," Box I 1965-58 \# I-35, Program and Media Studies, Record Group 306, National Archives and Records Administration, Washington, D. C. [hereafter NARA]. La expansión de programas culturales oficiales y privados en el extranjero coincidió con-y en parte respondió a — la fuerte señal del Presidente Eisenhower al sector privado en su "Baylor Proposals" de 1956, y en el posterior involucramiento de Ford Foundation en la diplomacia cultural.

8 Rosenberg, Spreading..., 7.

9 Marcos Cueto, Missionaries of Science (Bloomington, 1994); Elizabeth Cobbs,_The Rich Neighbor Policy (New Haven, 1992); Gerald Haines, The Americanization of Brazil (Wilmington, 1989).

10 "A Comparative Study of the Audience for Mass Media in Three Latin American Capitals: I053/ International Public Opinion Research at Chile, Panama, Mexico," Box 3 "Special Reports, 1953-63," Record Group 306, NARA.

I I Ver nota 6. In addition, Akira Iriye, "Culture and Power: International Relations and Intercultural Relations," Diplomatic History 10 (Spring 1979) I I 5-28; Mertin W. Sampson III, "Cultural Influences on Foreign Policy," Charles F. Hermann, Charles W. Kegley, Jr., James N. Rosenau, eds., New Directions in the Study of Foreign Policy (Boston, 1987).

12 Jessica C. E. Gienow Hecht, "Art is Democracy and Democracy is Art: Culture, Propaganda, and the Neue Zeitung in Germany, 1944-1947," Diplomatic History 23 (Winter 1999); Rob Kroes, If You've Seen 
One You've seen the Mall: Europeans and American Mass Culture (Urbana: University of Illinois Press, 1996); Reinhold Wagnleitner, The Coca-Colonization of the Cold War: The United States Cultural Mission in Austria after the Second World War (Chapel Hill: University of North carolina Press, 1994); Richard Kuisel, Seducing the French: The Dilemma of Americanization (Berkeley: University of California Press, 1993); Victoria De Grazia, "Mass Culture and Sovereignty: The American Challenge to European Cinemas, 1920-1960," Journal of Modern History 6 I (March 1989).

13 Max Weber, The Protestant Ethic and the Spirit of Capitalism (New York: Charles Scribner's Sons, 1958) I3.

I 4 Roberto Russell, "La globalización: situación y proceso," Ciclos, Año VIII, vol.VIII, número especial I415, ler. Semestre 1998, 43.

15. Cf. James Rosenau, "The Complexities and Contradictions of Globalization," Current History 96, n. 613 (November 1997).

16 Patrick Wolfe, "Imperialism and History: A Century of Theory, from Marx to Postcolonialism," American Historical Review 102, 2 (April 1997) 388-420; Frederick Coper, Alen F. Isaacman, Florencia E. Mallon, William Roseberry and Steve J. Stern, Confronting Historical Paradigms: peasants, Labor, and the Capitalist World System in Africa and Latin America (Madison: University of Wisconsin Press, 1993); Bill Ashcroft, Gareth Griffiths and Helen Tiffin, eds., The Empire Writes Back: Theory and Practice in Post-colonial Literature (London: Routledge, 1989); Patrick Williams and Laura Chrisman, eds.,_Colonial Discourse and Post Colonial Theory: A Reader (London: harvester Wheatsheaf, 1993).

17 Discusiones interesantes sobre el tema pueden encontrarse en Michael S. Hunt, "The Long Crisis in Diplomatic History: Coming to Closure," Diplomatic History 16 (Winter 1992) I I 5- I 40; Michael J. Hogan and Thomas G. Paterson, eds., Explaining the History of American Foreign Relations (New York: Cambridge University Press, 199I); Charles S. Maier, "Marking Time: The Historiography of International Relations," Michael Kammen, ed., The Past Before Us: Contemporary Historical Writing in the United States (Ithaca: Cornell University Press, 1980).

I 8 Cf. Amy Kaplan and Donald Pease, eds., Cultures of United States Imperialism (Durham; Duke University Press, 1996).

19 Wiliam Connolly, The Ethos of Pluralization Minneapolis: University of Minnesota Press, 1995), 14. Citado en su valioso análisis de la aplicación de paradigmas post-modernos al campo tradicional de las Relaciones Internacionales por Frank Ninkovich, "No Post-Mortems for Postmodernism," Diplomatic History 22 (Summer 1998) 45 I-466.

20 William Roseberry, Anthropologies and Histories: Essays in Culture, History and Political Economy (New Brunswick: Rutgers University Press, 1989) 42.

21 Gilbert M. Joseph, "Close Encounters: Toward a New Cultural History of U. S.- Latin American Relations," in Gilbert M. Joseph, Catherine LeGrand and Ricardo D. Salvatore, eds., Close Encounters of Empire: Writing the Cultural History of U. S. - Latin American Relations (Durham: Duke University Press, 1998) 8.

22. Gilbert M. Joseph, Catherine LeGrand and Ricardo D. Salvatore, eds., Close Encounters of Empire... es una colección de ensayos sobre relaciones interamericanas que logra establecer un diálogo, a veces implícitamente, entre diversos campos teoréticos y acercamientos paradigmáticos. Los artículos buscan superar, generalmente con éxito, algunas polarizaciones teoréticas y metodológicas como los pares economía política/ cultura; metanarrativas/minihistorias; sujetos fluidos/complejos totalizadores; fronteras/ cuerpos; imperialismo/ subalternidad.

23 Gilbert M. Joseph, "Close Encounters...," 5. El autor toma prestado el término de Mary Louise Pratt, Imperial Eyes: Travel, Writing and Transculturation (London: Routledge, 1992) 6-7; and Arts of the Contact Zone, Profession 9I ( I991) 33-39.

24 Gilbert M. Joseph, "Close Encounters...," 8.

25 Sobre el período post-Peronista en Argentina, entre otros, Celia Szusterman, Frondizi and the Politics of Developmentalism in Argentina (Pittsburgh, 1993); Oscar Terán, Nuestros años sesentas (Buenos Aires (1991), Silvia Sigal, Intelectuales y poder en la década del sesenta (Buenos Aires, 1991); Daniel James, Resistance and Integration (Cambridge, 1988); Gary Wynia, Argentina: Illusions and Reality (New York, 1986); Guillermo O’Donnell, El Estado burocrático- autoritario (Buenos Aires, 1981). 
26 Ver el trabajo del autor "Selling Americanism Abroad: ..." Siguiendo la teoría de Pierre Bourdieu sobre la dinámica de los campos culturales, se demuestra cómo el grupo de intelectuales asociados con programas de EE.UU. desafía con éxito a los grupos establecidos dentro de diferentes disciplinas. De allí nace la sugerencia de que la confrontación siguiente dentro del campo de cada disciplina respondió más a temas personales/ profesionales que a temas paradigmáticos/ideológicos. Ver Pierre Bourdieu, "The Field of Cultural Production, or: The Economic World Reversed," Poetics I 2 ( I 983) 3 I I-3 I 4, 330-332; "The Social Space and the Genesis of Groups," Theory and Society I 4 (1985) 723-744, 735-737; y "Les fractions de la classe dominante et les modes d'appropriation de l'oeuvre d'art," Social Science Information XIII, n. 3 (Juin 1974) 7-32.

27 Entrevista con el ex Rector de la Universidad de Buenos Aires, Dr. Julio Olivera, Buenos Aires, 7-29-96. También Revista Latinoamericana de Sociología, Buenos Aires, ICSSR, Instituto Di Tella, Año I, n. I ( 965 ). 28 Atilio Reggiani, "El intercambio cultural: un aspecto de lo revolucionario," Cuadernos de Cultura, 32 (Noviembre 1957) 159-166.

29 Crisólogo Gatica, Imperialismo y educación en America Latina," Cuadernos de Cultura 6I, (Enero/ Febrero 1963) 43-62.

30 Memo, "Social Science Program, Argentina" a Dr. Reynold Carlson, Buenos Aires, from Nita Manitzas, |2-3|-7|. Ford Foundation Archives, New York City [hereafter FFA].

3 I Kenneth W. Thompson, Vice-President, Rockefeller Foundation, Interview with J. P. Harrison, Assistant Director for Humanities, I0- |8-61, Folder 82, Box 10, Series 30 I R, Record Group I.2, RAC.

32 John P. Harrison, "Trip to Latin America: John Finan on José Luis Romero," 5-20-58, Folder 8I, Box I0, Series 30 I R, Record Group I.2, RAC.

33 "Entrevista con José Luis Romero, 12-12-56," y Official Indices Check, Folder 85 "José Luis Romero in the U. S. , 1956," Box 10, Series 301, Record Group I.2, RAC. Durante una reunión privada con J. P. Harrison, Romero explicaba que, habiendo sido miembro del anti-comunista pero moderado Partido Socialista, compartía las dificultades de muchos argentinos en "comprender las actividades de la 'New Frontier' en América Latina." "JPH Trip Diary," Folder 82 "UBA and American Foundations," Box 10, Series 30I R, Record Group I.2, RAC.

34. Kenneth W. Thompson, Vice-President, Rockefeller Foundation, Interview with J. P. Harrison... 35 Pierre Harmel, "Perspectivas sociales y económicas del crecimiento de la enseñanza," Criterio, Año XXXI, n. 1325 (2-12-59) 86.

36 Torcuato Di Tella, "Tensiones sociales en los países de la periferia;" Kalman Silvert, "Nacionalismo y desarrollo," Revista de la Universidad de Buenos Aires, V Epoca, Año VI, n. I, (Enero- Marzo I96 I).

37 . Encuestas y entrevistas: "Los subsidios," Revista de la Universidad de Buenos Aires, V Epoca, Año VIII, n. 3-4 (Julio- Diciembre 1963) 54I-53.

38 Carta, Al Wolf a Bernardo Houssay, President, CONICyT, I-7-60, Folder "Ford Foundation 1959," Archivos del CONICET, Buenos Aires.

39 L. C. D. (sic), "Interview with Enrique Oteiza," 10-26-6I, Folder 82, Box 10, Series 30 I R, Record Group I.2, RAC.

40 . Juan Carlos Torre, [Robert Lynd and the Critique of Sociology," Pasado y Presente (Córdoba), I, n. 2/3 (7-12-63) 194.

4I "Un juicio sobre C. Wright Mills," Cuadernos de Cultura 64 (Julio/ Agosto 1967) 94-95.

42 Memo, "Report on Argentina," de Morris A. Horowitz a Verne A. Atwater, representante de Ford Foundation en Argentina y Chile, 8-27-62. FFA, PA 62-26. Dr. Horowitz' Folder. Esta visión contrasta con el memo de Enrique Oteiza, Director Ejecutivo del ITDT' a Javier Villanueva, Director del CER, 7- I265, Folder "Correspondencia 1960/79," Box CIE Dirección 4.a, Archivos del Instituto Torcuato Di Tella, Buenos Aires [desde ahora ITDT].

43 "Guillermo Edelberg, 1965," Box 4-C (Dirección), ITDT.

44 Carta, Dr, H. J. A. Rimoldi a Raúl Hinsch, Pro-secretario CONICyT, 5-8-6I. Folder "Centro de Psicología Industrial,"Box I, CONICET.

45 "Proposal for the Creation of a Center for Government Studies for Planning Operations." Enrique 


\section{REVISTA ESBOÇOS N 20 - UFSC}

Oteiza a Al Wolf, Director, Overseas Devolpment Program, Ford Foundation, 5-|8-6|, Box "Ford Foundation," ITDT.

46 "Informe sobre el estudio de Aníbal Pinto y Osvaldo Sunkel," Box "ClE: Estudios de Post-Grado," ITDT.

47 Discurso de Enrique Oteiza en la Escuela de Ciencias Médicas, Universidad de Buenos Aires, 7-3-64. Box "Radio," ITDT.

48 R. K. D. (sic), Entrevista con Kingsley Davis, Guido Di Tella y Gino Germani, New York, 1-29-62, Folder 87 "Torcuato Di Tella Institute," Box 10, Series 30 I, Record Group I.2, RAC.

49 Entrevista con Torcuato Di Tella, Jr., Buenos Aires, 7-19-96.

50 Entrevista con Enrique Oteiza, Buenos Aires, 8- |4/I5-96.

5I "Alonso-Rasmussen-Silvert-Urquidi Visisting Team to the Di Tella Institute," 8- II/I5-69, FFA, PA 68572, 5.

52 Memo, "Conversation with Gilda Romero Brest," to Robert G. Myers, New York, from Jeffrey M. Puryear, 9-6-74, FFA, PA 67-286.

53 Memo, "Social Science Program...," I.

54 Entrevista con Torcuato Di Tella, Jr.,...

55 Entrevista con Enrique Oteiza... Entrevista con Dr. José Nun, ex investigador del ITDT, Buenos Aires, 10-14-96. 